Meeting Report

\title{
TEDD Annual Meeting 2020: 10 th Anniversary - 10 Years of Innovation in Tissue Engineering
}

doi:10.14573/altex.2012151

As every year, we dedicated the TEDD (Tissue Engineering for Drug Development and Substance Testing) Annual Meeting 2020, held on October 22, to cutting edge academic and industrial research. This year, we focused on microphysiological systems (MPS) and their application in different fields, including research on COVID-19 and the development of in vitro disease and infection models. The online event featured scientific presentations and allowed participants to network with unique matchmaking tools and virtual 1:1 meetings. Additionally, we provided the companies with the possibility to showcase their services and products in online exhibition sessions during the virtual lunch break.

The TEDD Annual Meeting 2020 celebrated ten years of the TEDD network. In 2010, TEDD, established by Prof Dr Ursula Graf-Hausner (ZHAW) and InSphero, was a pioneer in the Swiss research landscape, and TEDD researchers and their partners are still at the forefront today. Ten years ago, organotypic cultures and microphysiological systems where mainly developed and used in academic settings. Nowadays, the different technologies, including bioprinting, are on the way to industrial implementation. Dr Markus Rimann, the current TEDD leader, was one of the first to introduce bioprinting in Switzerland.

The symposium presentations covered a broad range of academic and industrial research. Dr Uwe Marx, scientific founder and CSO of TissUse, dedicated to the development of human organand body-on-a-chip systems for drug testing and precision medicine approaches, opened the scientific program with a keynote. He summarized the findings of the second stakeholder workshop on "Biology-Inspired Microphysiological Systems (MPS) to Advance Patient Benefit and Animal Welfare in Drug Development" (doi:10.14573/altex.2001241). Dr Marx talked about the status of industrial adoption and regulatory acceptance for candidate decision-making with MPS. MPS have proven to be powerful tools for recreating human tissue- and organ-like functions at research level. The research provides the basis for the establishment of qualified preclinical assays with improved predictive power. However, industrial adoption of MPS and respective assays is progressing slowly due to their complexity. In the first part, Dr Marx presented the status quo of MPS development and examples of industrial adoption of single- and two-organ chip solutions. He talked about the underlying universal microfluidic Multi-Organ-Chip (MOC) platform, which is the size of a microscopic slide, integrates an on-chip micro-pump, and is capable of interconnecting different organ equivalents. His team has established sixteen single organ equivalents and twelve organ combinations and tested them for stable long-term crosstalk. In the second part of the presentation, Dr Marx focused on the challenges of translating a MOC-based combination of four human organ equivalents into a commercially useful tool for pharmacokinetic profiling and toxicity testing of drug candidates. The four-organ tissue chip combines intestine, liver, and kidney equivalents for adsorption, metabolism, and excretion, and a neuronal compartment for extended toxicity testing, respectively. Dr Marx discussed issues to ensure long-term performance and industrial acceptance of such complex MPS, such as design criteria, tissue supply, and on-chip tissue homeostasis. Finally, Dr Marx provided a roadmap towards on-chip patient models, which bear the potential of a paradigm shift in drug development and clinical research.

Dr Samuel Constant, co-founder and CEO of Epithelix, a Swiss biotech company specialized in tissue engineering, talked about 3D human airway epithelial models to study SARS-CoV-2 pathogenesis. The recent outbreak of SARS-CoV-2, leading to the disease COVID-19, is a significant threat to human beings. The respiratory system is the first and leading entry portal of SARS$\mathrm{CoV}-2$, which initially and principally infects the airway epithelia. It then gradually propagates to other human organs, causing symptoms such as loss of taste, fever, dry cough, fatigue, diarrhea, conjunctivitis, pneumonia, and respiratory failure. Vaccination and new antiviral drugs are needed. Since the first step of SARSCoV-2 infection takes place in airway epithelial cells, it is logical to use 3D airway epithelial models as drug testing platforms. Epithelix offers standardized air-liquid interface 3D human airway epithelial cultures from nasal or bronchial (MucilAir ${ }^{\mathrm{TM}}$ ) and small-airway (SmallAir ${ }^{\mathrm{TM}}$ ) origins. These epithelial models closely mimic the morphology and function of the native tissues, such as cilia formation and beating, mucus production and secretion, mucociliary clearance, and secretion of antiviral molecules. Epithelix and their collaborators have used these models successfully for the development of antivirals against influenza, rhinoviruses, respiratory syncytial virus, and others. In his talk, Dr Constant highlighted how these models could be used to characterize viral infection kinetics, tissue-level tropism, and transcriptional immune signatures induced by SARS-CoV-2. He pointed out the relevance of these models for preclinical evaluation of antiviral candidates in the context of repositioning of marketed drugs or evaluation of novel therapies and drug combinations delivered systemically or through aerosol therapy.

In the following talk, Dr Sebastien Mosser, COO at Neurix, a Swiss company that develops in vitro neuronal tissues for compound screening and neuroscience research, described multiple facets of cerebral organoids for scientific research and drug testing. $90 \%$ of new drug compounds fail in clinical trials, which causes a massive waste of time and money by pharmaceutical companies. The great majority of failures is due to drug toxicity or lack of efficiency in humans, suggesting that both in vitro and in vivo preclinical tests are poorly relevant to human physiology. Indeed, animal models are not humans, and cells cultivated in vitro 
on flat surfaces weakly reflect organs. To mimic the complexity and architecture of the human brain as closely as possible, Neurix manufactures cerebral organoids as an alternative to animal experimentation. Two types of organoids are derived from human pluripotent stem cells: Neurospheres, which are well suited for high throughput screening required by pharmaceutical companies, and the Minibrain ${ }^{\circledR}$, which is a larger organoid adapted to neuroscience research and lead compound testing. Dr Mosser presented what the company provides in terms of drug and treatment testing services to assess brain cancer, gene and cell therapy efficiency, neurotoxicity, and custom research projects. He showed how they are optimizing their system by the addition of immune cells to model neuroinflammatory phenotypes more efficiently.

Dr Nuria Montserrat from the Institute for Bioengineering of Catalonia, Spain presented engineering solutions for human pluripotent stem cell (hPSC) differentiation, from kidney organoid derivation to disease modelling applications. Since 2008, Dr Montserrat has worked on the development of strategies for the generation and banking of new induced pluripotent stem cells (iPSCs) and new approaches to reprogram somatic cells and further differentiate iPSCs into mesoderm-like tissues, including cardiomyocytes and renal progenitor cells. The generation of $\mathrm{hP}-$ SC-derived organoids is one of the most significant scientific advances in regenerative medicine. Recently, her group generated kidney organoids that transcriptomically matched second-trimester human fetal kidneys. This was achieved by lengthening the culture time of hPSCs in a three-dimensional microenvironment in the presence of defined renal inductive signals. Furthermore, Dr Montserrat developed a transplantation method that exploits the intrinsic properties of the chick chorioallantoic membrane (CAM) to recreate a soft in vivo microenvironment for organoid growth and differentiation, including in vivo vascularization. Next, through bioengineering, they have mimicked the stiffness of the chick CAM by fabricating compliant hydrogels. Their recent findings include exploiting the kidney organoid platform with CRISPR/Cas9 technology for applications in disease modelling and the study of morphogenesis. Dr Montserrat discussed how these findings have resulted in the application of procedures for kidney organoid derivation in the study of SARS-CoV-2 infection and the identification of soluble recombinant ACE2 (receptor for virus-cell entry) as a putative treatment to reduce SARS-CoV-2 infection in human-engineered microtissues. Dr Montserrat also presented recent findings on the derivation of hydrogels from decellularized human and porcine kidneys and their utility in kidney organoid engineering.

The next speaker was Dr Tina Bürki-Thurnherr from the Swiss Federal Laboratories for Materials Science and Technologies (Empa), Switzerland. She presented human health risks of nanomaterials at and beyond protective biological barriers, and how they exhibit unique properties, enabling a wealth of novel applications in many fields. Dr Bürki aims at a comprehensive understanding of potential human health risks as a prerequisite to the safe and sustainable use of these promising materials. Biological barriers perform essential protective and supportive functions and are thus po- tentially prone to damage from exposure to nanomaterials. In her presentation, she provided new insights on the impact of repeated graphene oxide exposure to healthy or Streptococcus pneumonia-infected reconstituted primary human bronchial epithelium. Furthermore, she presented the development of a new placenta-on-chip to assess potential fetotoxic risks of nanomaterials. With this research, her group aims to support the safe development of nanomaterials for industrial, commercial, and medical applications.

As the last scientific talk of the day, Dr Jochen Kühnl from Beiersdorf AG, Germany, presented a case study to characterize exposure scenario-dependent chemical metabolism in a MPS combining skin and liver organ models. They performed case studies within Cosmetics Europe's Long Range Science Strategy (LRSS), which aims to establish new solutions and to test options to enable next-generation risk assessment. Combinations of 3D tissue models in MPS are promising approaches to provide relevant data for risk assessment. Dr Kühnl presented examples of recent case studies utilizing a microfluidic platform technology (HUMIMIC Chip2 model), incorporating skin (EpiDerm ${ }^{\mathrm{TM}}$ ) models and liver spheroids derived from HepaRG and stellate cells to investigate the robustness of the system and to test exposure scenario-dependent differences in the bioavailability and metabolic fate of chemicals, as well as tissue responses. EpiDerm ${ }^{\mathrm{TM}}$ models and liver spheroids were well maintained over six days, as indicated by metabolic analysis and viability markers. Case study chemicals included hyperforin (a component of St. John's Wort) and 4-amino-2-hydroxytoluene (AHT, an aromatic amine hair dye). Scientists chose AHT to determine whether the Chip2 model could emulate the first-pass effect in the skin observed in in vivo studies in rats. The results indicated route-specific differences in bioavailability due to the skin barrier function and showed excellent intra- and inter-laboratory reproducibility in all endpoints measured. He concluded that HUMIMIC technology could provide information on route-dependent differences in parent and metabolite kinetics, as well as corresponding effects on tissues that may be relevant to risk assessment.

In addition to the scientific symposium, twelve TEDD Partner companies presented their science, services and products during the virtual lunch break sessions chaired by Dr Markus Rimann, Prof Michael Raghunath, Dr Jens M. Kelm and Dr Bruno Fillipi. Finally, Dr Markus Rimann closed the meeting by presenting future ideas of the TEDD network, which will focus on industry implementation of the different technologies as well as enlarging the network to create cross-industrial benefit.

\section{Katarzyna S. Kopanskal and Markus Rimann ${ }^{1,2}$}

${ }^{1}$ Competence Centre TEDD, Institute of Chemistry and Biotechnology (ICBT), ZHAW Zurich University of Applied Sciences, Wädenswil, Switzerland; ${ }^{2}$ Centre for Cell Biology \& Tissue Engineering, Institute of Chemistry and Biotechnology (ICBT), ZHAW Zurich University of Applied Sciences, Wädenswil, Switzerland

(katarzyna.kopanska@zhaw.ch) 